\title{
Learning Character Recognition with Graph-based Privileged Information
}

\author{
Florian Westphal*, Niklas Lavesson*†, Håkan Grahn* \\ * Department of Computer Science, Blekinge Institute of Technology, Karlskrona, Sweden \\ \{florian.westphal,hakan.grahn\}@bth.se \\ ${ }^{\dagger}$ Computer Science and Informatics, Jönköping University, Jönköping, Sweden \\ niklas.lavesson@ju.se
}

\begin{abstract}
This paper proposes a pre-training method for neural network-based character recognizers to reduce the required amount of training data, and thus the human labeling effort. The proposed method transfers knowledge about the similarities between graph representations of characters to the recognizer by training to predict the graph edit distance. We show that convolutional neural networks trained with this method outperform traditional supervised learning if only ten or less labeled images per class are available. Furthermore, we show that our approach performs up to $33 \%$ better than a graph edit distance based recognition approach, even if only one labeled image per class is available.
\end{abstract}

Keywords-character recognition; learning using privileged information; graph matching; convolutional neural networks

\section{INTRODUCTION}

In recent years, machine learning and particularly deep learning approaches have become popular as solution for document analysis and recognition problems, due to their good performance. However, achieving this good performance requires large amounts of training data. This makes deep learning approaches difficult to use for companies and government institutions, which possess large amounts of diverse document image collections, since they may have to invest into creating labeled subsets for each of their document collections. Therefore, it is important to reduce the amount of labeled data needed for these approaches.

In this paper, we aim to achieve this goal for character recognition with the help of a learning using privileged information (LUPI) based approach [1], [2]. In LUPI, a different representation of the training data is provided as privileged information, which tends to be harder to acquire, but from which it is easier to learn the actual task. Thus, privileged information is used to help learning the task from fewer training samples, while not being available during normal operation. Character recognition was chosen to allow a thorough exploration of possible approaches, while making it likely that obtained conclusions can be transferred to similar document analysis tasks, such as word spotting [3].

The main contribution of this paper is the proposal to use graphs representing the character to be recognized, as shown in Fig. 1, as privileged information in a LUPI based approach. This is motivated by the fact that graph matching is known to outperform learning based approaches, if only

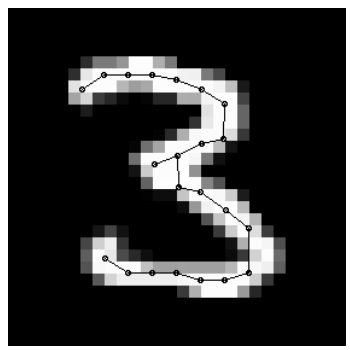

(a) MNIST Digit

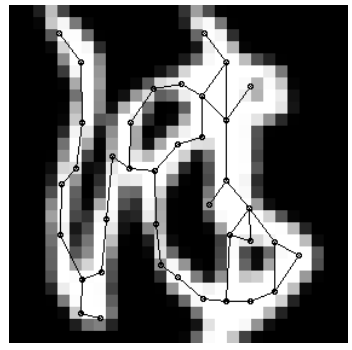

(b) KMNIST Character
Figure 1. Graph for a digit from the MNIST data set [5] and one character from the Kuzushiji-MNIST (KMNIST) data set [6].

few training samples are available [4]. We transfer the knowledge about the similarity between different character images, as represented by the Graph Edit Distance (GED) between their corresponding graphs, to a Convolutional Neural Network (CNN) by learning to predict the GED. In this way, access to the privileged information, i.e., the character graphs and their respective GEDs, is not necessary in normal operation. While this information can be generated automatically, it is beneficial not to rely on it during operation, since it slows down the recognition speed.

Given that the GED can be learned sufficiently well by the $\mathrm{CNN}$, it is reasonable to assume that it will perform equally well or better in terms of character recognition performance than a GED based character recognizer, even if only few labeled instances are provided for training. This implies that a CNN trained in this way will outperform a CNN trained using a standard supervised learning procedure given only few training samples. However, it is known that CNN based approaches outperform GED based approaches given sufficient training data [4]. Therefore, it is reasonable to assume that the proposed approach will loose its advantage over standard supervised learning if just enough training data is provided. This raises the question: How much is enough?

Our results show that for a simple digit recognition task our proposed approach performs better than a standard supervised learning procedure if only five or less instances per class can be provided. For more complex handwritten Japanese characters, this number rises to ten labeled instances per class. After the respective threshold is passed, 
our approach performs similar to supervised learning. Furthermore, we show that our approach performs statistically significantly better than the GED based approach on both data sets, even if only one training sample per class is given.

\section{BACKGROUND AND RELATED WORK}

\section{A. Learning Using Privileged Information}

Learning using privileged information (LUPI) is a learning paradigm, which aims to reduce the amount of training examples needed by an algorithm to learn a certain concept [1], [2]. This is accomplished under LUPI by introducing an intelligent teacher, which provides additional information about the available training examples. Thus, the training set consists of triplets $\left(x_{i}, x_{i}^{*}, y_{i}\right)$ with $x_{i} \in X$, $x_{i}^{*} \in X^{*}$ and $y_{i} \in Y$. Here, $X$ is the set of instances in the feature representation, which will be available at test time, $X^{*}$ is the set of instances in the privileged feature representation as given by the intelligent teacher and $Y$ is the set of instance labels. It is important to note that the set $X^{*}$ is available during training time, but not during testing.

In order to make use of the given privileged information, its use can either be integrated into the algorithm's learning process directly or it can be used in a framework of knowledge transfer [7]. In the latter case, the privileged information is used to train a separate learner $f^{\prime}: X^{*} \rightarrow Y$, which then has to transfer its knowledge to the actual learner $f: X \rightarrow Y$. In this paper, we follow this second approach.

\section{B. Graph Matching}

Graph matching is a common technique in pattern recognition [8], [9], which has been applied in document analysis to keyword spotting [4] and signature verification [10]. The main idea is to define a graph, which traces the lines of a word or a signature with its edges, as shown in Fig. 1 for characters. This graph can then be used to match against other word graphs by calculating the Graph Edit Distance (GED) between them. This distance is based on the amount of node and edge insertions, deletions and substitutions required to transform one graph into the other. Thus, graphs of the same or similar words will have small GEDs. While computing the exact GED is an NP-hard problem, there are efficient approximations, such as the Hausdorff Edit Distance (HED) [11], which is used here.

\section{Siamese Networks}

A Siamese network is a neural network architecture, which was initially proposed for fingerprint matching [12] and signature verification [13]. The main idea is to train two CNNs with tied weights as feature extractors for pairs of images. The distance between the extracted feature vectors is assessed and used as base for predicting the probability of whether or not the two given images are similar to each other. By learning this probability, the $\mathrm{CNN}$ feature extractors learn to extract an image's feature vector, which is close to the feature vectors of similar images and farther away from feature vectors of dissimilar images.

\section{Related Work}

Different LUPI approaches have been proposed for digit recognition. For example, Vapnik and Vashist [2] have used poetic descriptions of digits from the MNIST data set [5] as privileged information to train an SVM+ classifier. Another approach has been taken by Lopez-Paz et al. [14], who use the original $28 \times 28$ pixel large MNIST digits as privileged information, while down-sampled images of size $7 \times 7$ serve as inputs to a multilayer perceptron classifier.

One broader area related to the overall aim of this paper is few-shot learning (FSL), which is concerned with approaches learning a new task from a small number of training samples. Based on the FSL classification by Wang et al. [15], our approach can be considered a model based FSL technique using task-specific embedding learning.

\section{LEARNING FROM PRIVILEGED INFORMATION}

\section{A. Graphs as Privileged Information}

In contrast to previous approaches, we are using graphs as privileged information. The main motivation for using this information for a knowledge transfer based LUPI approach is the high correspondence between the actual instances, i.e., the character images, and their respective graphs. Thus, transferring the knowledge about similarities between different character instances, as expressed by the GED, is likely to teach the actual learner $f$ to extract relevant features from the images. These features should allow $f$ to learn the recognition task from only a few labeled images.

Here, we use the projection profile based graph extraction algorithm by Stauffer et al. [16] as intelligent teacher, which provides the privileged information. This method extracts the graphs automatically from a given image using vertical and horizontal projection profiles. Fig. 1 shows two example images and their respective extracted graphs.

To compute the graph edit distance between the defined graphs, we use the Hausdorff Edit Distance (HED) [11]. As proposed by Stauffer et al. [4], we standardize the node locations of each graph by subtracting their mean and dividing them by their standard deviation. Similarly, we use a constant $\operatorname{cost} \tau_{v}$ for node deletions and insertions and a constant cost $\tau_{e}$ for edge deletions and insertions. Furthermore, we follow their approach by using a Euclidean distance based cost for node substitutions and by using an $\alpha$ and $\beta$ parameter to control the importance of edge compared to node operations, as well as the importance of deviations in horizontal or vertical direction respectively.

\section{B. Knowledge Transfer Using Siamese Networks}

We transfer the knowledge about similarities and differences of training images from the privileged information domain to a CNN based classifier by training a Siamese 


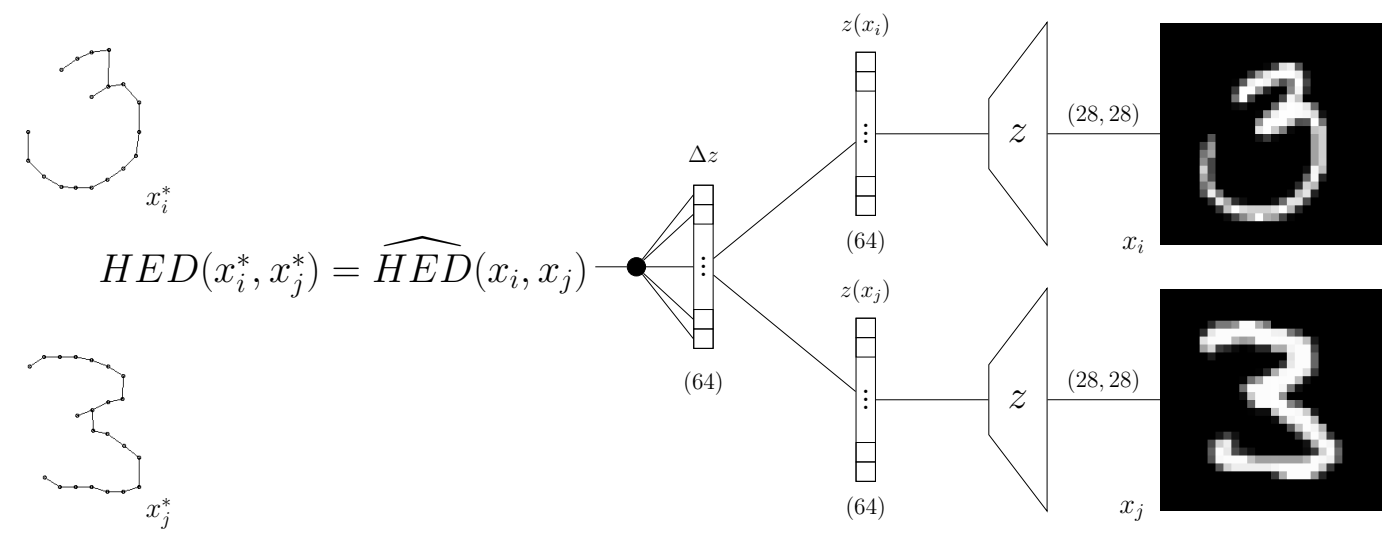

Figure 2. Illustration of the Siamese network architecture to learn the approximate graph edit distance $\widehat{H E D}\left(x_{i}, x_{j}\right)$ for the images $x_{i}$ and $x_{j}$ from the Hausdorff Edit Distance between their corresponding graphs $x_{i}^{*}$ and $x_{j}^{*}$.

network to predict the unnormalized HED, as illustrated in Fig. 2. As shown in the figure, the Siamese network receives two images, $x_{i}$ and $x_{j}$, as input and is trained to predict the HED between the corresponding graphs, $x_{i}^{*}$ and $x_{j}^{*}$. Each image is processed by a ResNet-20 [17] type CNN base $z$, which extracts a 64-dimensional feature vector per image. The HED is predicted by taking the weighted sum over the absolute differences between the two feature vectors and the network is trained by minimizing the mean squared error.

This training procedure forces $z$ to learn to extract 64 relevant features from a given image to map this image into a 64-dimensional embedding space, in which the distances between points reproduce the edit distances between the respective graphs. Since this feature extraction is learned, in contrast to the handcrafted nature of the graph extraction and edit distance computation, it is likely that a character recognizer based on $z$ will perform as good or better than an HED based recognizer. This implies that a $z$ based recognizer will perform better than a CNN based recognizer trained using standard supervised learning for small training set sizes. However, this advantage is limited by the HED's usefulness for character recognition and is thus likely to decrease the more training data becomes available.

One challenge for the proposed approach is that learning the HED is presumably harder than learning to recognize characters, since the edit distance is a more complex concept than the concept of a character class. Hence, more training data should be required to learn the HED than to learn the actual recognition task. However, this does not increase the human labeling effort, since the graph extraction and the HED computation do not require labeled data. Thus, we pre-train the ResNet-20 CNN base $z$ on a large number of images and their corresponding HEDs. This pre-trained base is then used as foundation for different recognizers to learn the actual recognition task from few labeled instances.

\section{EXPERIMENT DESIGN}

\section{A. Experiment Setup}

All experiments were conducted on a computer with an Intel i9-7900X CPU @ $3.3 \mathrm{GHz}, 32$ GB DDR4 RAM and two Nvidia GeForce GTX 1080 Ti. The projection profile based graph extraction has been implemented ${ }^{1}$ based on the description by Stauffer et al. [16]. The GMatch4Py ${ }^{2}$ implementation of the HED has been modified to match the description by Stauffer et al. [4]. Our approach for learning the HED and all evaluated CNN based character recognizers have been implemented in Keras ${ }^{3}$ version 2.2.4 based on the example ResNet implementation provided by Keras.

We evaluate our proposed approach on the MNIST [5] and KMNIST [6] data set. While the MNIST data set consist of modern handwritten digits with ten classes, the KMNIST data set consists of historical handwritten Japanese characters with ten character classes. Images from both data sets are shown in Fig. 1. Both data sets provide 60000 labeled training images and 10000 labeled test images.

In all experiments, we measure the performance of the evaluated digit recognizers in terms of accuracy. The accuracy $a c c$ is defined as follows:

$$
a c c=\frac{1}{|T e|} \sum_{x \in T e} I[c(x)=\hat{c}(x)]
$$

Here, $T e$ denotes the test data set, $I[\cdot]$ is the indicator function, and $c(x)$ and $\hat{c}(x)$ are the actual and the predicted class label of $x$ respectively.

\section{B. Evaluated Character Recognizers}

We evaluate our proposed training approach by comparing following digit recognizers.

\footnotetext{
${ }^{1}$ All implementations used in this paper, as well as the raw data are available at https://github.com/FlorianWestphal/ICDAR2019.

${ }^{2}$ https://github.com/Jacobe2169/GMatch4py

${ }^{3}$ https://keras.io
} 
$H E D_{k N N}$ : For this recognizer, we use the HED between all training graphs and the extracted character graph to label. The character class is determined by the extracted graph's $k$ nearest neighbors $(\mathrm{kNN})$ in terms of HED.

$\widehat{H E D_{k N N}}$ : This recognizer also uses a kNN approach for labeling the test images. However, in this case the 64-dimensional feature vectors, extracted by the pre-trained $\mathrm{CNN}$ base $z$, are used to determine the Euclidean distance between the train and test samples. Thus, this recognizer is expected to be most similar in performance to $H E D_{k N N}$.

$\widehat{H E D_{N N}}$ : Similar to $\widehat{H E D} D_{k N N}$, this recognizer uses the feature vectors extracted by the CNN base $z$, but uses them as input to a fully connected neural network layer with a softmax activation function to perform the classification.

$\widehat{H E D} D_{N N_{+}}$: This recognizer adds a fully connected layer with softmax activation function on top of $z$ and trains the entire CNN using the provided training samples.

$S$ : This recognizer uses the same network architecture as $\widehat{H E D} D_{N N+}$. However, it is trained from scratch with standard supervised learning using only the provided training data and no HED based pre-training.

\section{Training and Evaluation Procedure}

Since the main aim of the proposed approach is to train a character recognizer with only few training samples, we create ten randomly sampled, balanced training sets of size 10 , 30, 50, 70, 100, 250, 500, 1000 and 2500 from the MNIST and KMNIST training sets respectively. Furthermore, we randomly select corresponding balanced validation data sets of 100 images each from the respective training sets. The validation data set size has been chosen to be 100 to reduce the overall required human labeling effort. Additionally, we create a randomly chosen, balanced subset of the MNIST and KMNIST test set containing 1500 images for evaluating the $H E D_{k N N}$ recognizer. This has been necessary to ensure reasonable execution times for the HED computation.

We tune the parameters of the used graph extraction method and the parameters for the HED computation based on the tuning procedure described by Stauffer et al. [4]. The tuning is performed only on the images of the respective validation data set. Then, we train the CNN base $z$ on 2500 images by learning to predict the corresponding HEDs, which were computed using the tuned parameters. This model is trained for 100 epochs and the final configuration is chosen based on its performance on a validation set containing 500 images. Here, a larger validation data set can be used, since the images do not need to be labeled.

The evaluated character recognizers are trained on the selected training sets containing at most 1000 images. Where applicable, the recognizer is trained for 50 epochs and the validation data set of 100 images is used to choose the final model. Data augmentation in form of moderate rotations and shifts has been performed when training the CNN base and the digit recognizers, when applicable.

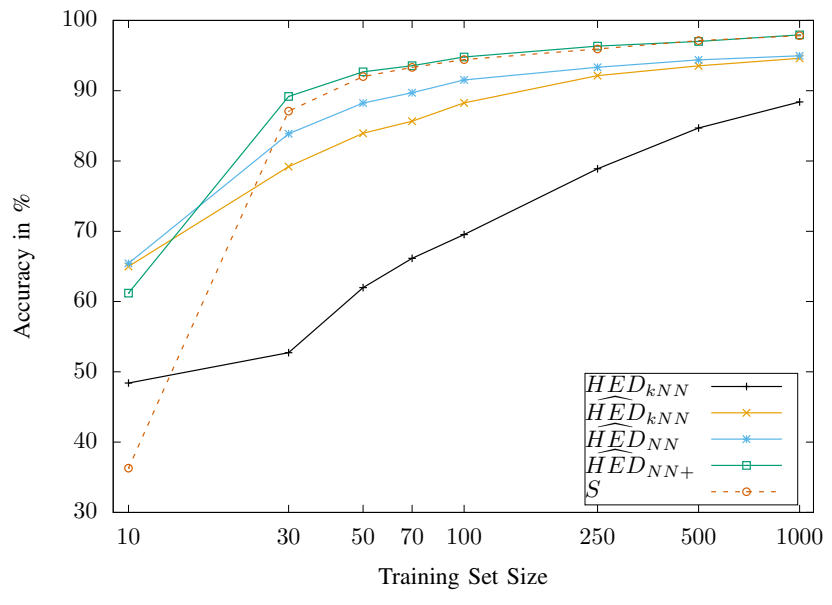

Figure 3. Average accuracy of the five evaluated recognizers on the MNIST data set [5] depending on the training set size.

We execute the described procedure for MNIST and KMNIST each ten times to obtain more reliable results.

\section{RESUlts AND ANALYSis}

\section{A. Comparison with Graph Matching}

In this section, we compare all five evaluated recognizers based on their performance on the randomly chosen subset of the MNIST and KMNIST test set containing 1500 images each. Fig. 3 and 4 show the average accuracies for all five recognizers depending on the training set size over ten randomly chosen training sets per training set size.

From Fig. 3, one can see that the HED based recognizer $H E D_{k N N}$ outperforms the CNN baseline classifier $S$ when only one labeled image per class is provided. However, $S$ outperforms $H E D_{k N N}$ as soon as more than one labeled image per class is available. Furthermore, one can see that the three recognizers based on the proposed pre-training strategy consistently outperform $H E D_{k N N}$. Similar observations can be made for the results obtained on the KMNIST data set, as shown in Fig. 4. Nevertheless, one difference with respect to $H E D_{k N N}$ is that it outperforms $\widehat{H E D} D_{N N}$ for a training set size of 1000 images. This is likely due to the inability of the trained feature extractor to learn feature representations for the Japanese characters, which are sufficiently separable for the fully connected neural network layer of $\widehat{H E D} D_{N N}$. However, the performance of $\widehat{H E D} D_{k N N}$, the recognizer most similar to $H E D_{k N N}$, shows that the extracted features are generally more suitable for character recognition than the HED.

The graph matching based $H E D_{k N N}$ should perform better than learning based approaches, if only few training samples are provided. Therefore, we analyze the recognition results for the five strategies, if only one image per class is provided, more closely. The box plots in Fig. 5 show that all recognizers based on the proposed pre-training 


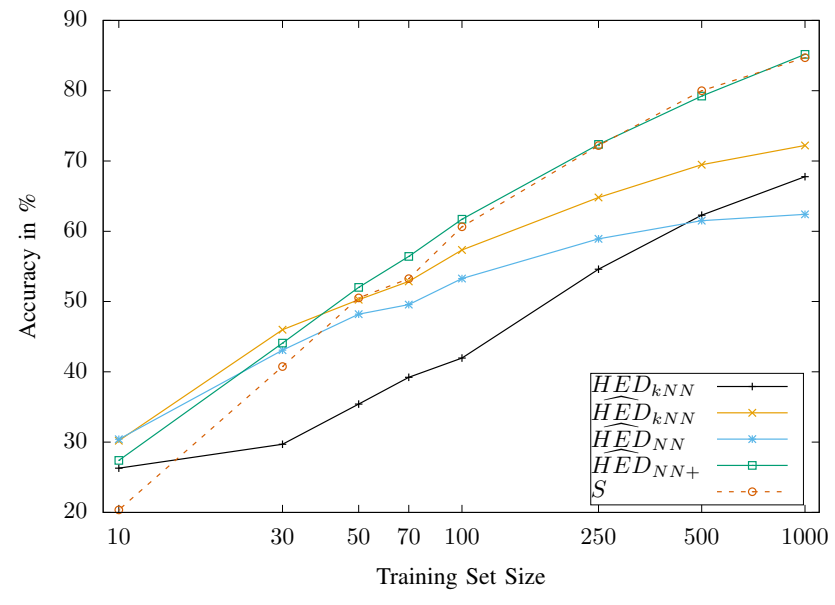

Figure 4. Average accuracy of the five evaluated recognizers on the KMNIST data set [6] depending on the training set size.

approach perform similarly to each other and better than both $H E D_{k N N}$ and $S$ on the MNIST data set. Furthermore, it appears from the figure that the proposed recognizers perform better than $S$ on the KMNIST data set.

In order to confirm the observed performance differences, we conduct a repeated measures analysis of variances (ANOVA), which indicates for both data sets statistically significant differences in mean accuracy at the $p<0.05$ level. For this reason, we identify the pairwise differences between the recognizers using Tukey's test as post hoc analysis. This analysis confirms the previously made observations about the performance differences at the $p<0.05$ level. Furthermore, it shows for the KMNIST data set that $H E D_{k N N}$ performs statistically significantly better

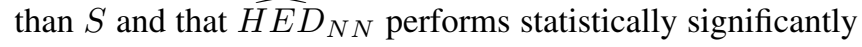
better than $H E D_{k N N}$. This analysis shows that the proposed learning based recognizers perform at least as well or better than the graph matching based approach even if only one labeled image per class is provided.

\section{B. Comparison with Standard Supervised Learning}

In this section, we compare the three HED pre-training based recognizers with the baseline $\mathrm{CNN}$ recognizer $S$ on the complete MNIST and KMNIST test set. We identify the training set size for which the proposed pre-training yields statistically significant benefits over standard supervised learning by performing a repeated measures ANOVA between the four recognizers for each training set size. Tukey's test is performed in cases where ANOVA identified statistically significant differences at the $p<0.05$ level. From these tests, one can see that the proposed pre-training results in statistically significant better accuracy than standard supervised learning only if at most 10 labeled images for MNIST and at most 30 labeled images for KMNIST are provided. However, when just comparing the achieved mean

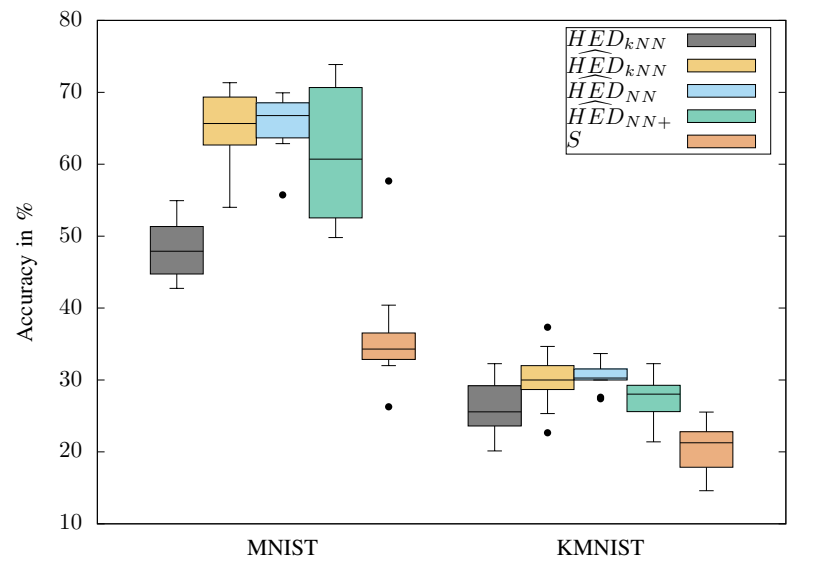

Figure 5. Accuracies of the five evaluated recognizers on the MNIST [5] and KMNIST [6] data set for a training set size of 10. Boxes show the first, second and third quartile; Whiskers indicate the lowest and highest value within the 1.5 interquartile range; Dots mark outliers outside this range.

Table I

RECOGNITION ACCURACY IN \% FOR THE EVALUATED RECOGNIZERS ON THE MNIST DATA SET [5] FOR DIFFERENT TRAINING SET SIZES.

\begin{tabular}{rcccc}
\hline & $\widehat{H E D} D_{k N N}$ & $\widehat{H E D} D_{N N}$ & $\widehat{H E D} D_{N N+}$ & $S$ \\
\hline 10 & $64.15 \pm 4.39$ & $\mathbf{6 6 . 1 1} \pm \mathbf{3 . 6 8}$ & $61.39 \pm 9.56$ & $35.85 \pm 7.38$ \\
30 & $79.17 \pm 3.93$ & $84.33 \pm 3.37$ & $\mathbf{8 9 . 4 2} \pm \mathbf{1 . 6 5}$ & $87.68 \pm 2.06$ \\
50 & $83.56 \pm 2.17$ & $88.11 \pm 1.55$ & $\mathbf{9 2 . 7 0} \pm \mathbf{1 . 0 4}$ & $91.85 \pm 1.16$ \\
70 & $86.49 \pm 1.69$ & $89.96 \pm 1.57$ & $\mathbf{9 3 . 6 8} \pm \mathbf{1 . 3 7}$ & $93.28 \pm 1.41$ \\
100 & $88.94 \pm 1.48$ & $91.49 \pm 1.17$ & $\mathbf{9 4 . 8 4} \pm \mathbf{0 . 8 0}$ & $94.57 \pm 0.66$ \\
250 & $92.65 \pm 1.31$ & $93.46 \pm 0.64$ & $\mathbf{9 6 . 2 0} \pm \mathbf{0 . 3 6}$ & $96.01 \pm 0.92$ \\
500 & $94.22 \pm 0.79$ & $94.51 \pm 0.45$ & $96.77 \pm 0.60$ & $\mathbf{9 6 . 9 9} \pm \mathbf{0 . 5 8}$ \\
1000 & $95.21 \pm 0.90$ & $94.94 \pm 0.34$ & $97.81 \pm 0.41$ & $\mathbf{9 7 . 8 3} \pm \mathbf{0 . 4 0}$ \\
\hline
\end{tabular}

accuracies of the different recognizers, as shown in Table I and II, it appears that the proposed pre-training can have a visible positive effect for training set sizes of up to 50 images for MNIST and 100 images for KMNIST.

Additionally, one can see from Table I and II, as well as from Fig. 3 and 4 , that the suitability of the extracted 64-dimensional feature vectors for recognition is limited by the usefulness of the HED. This is evidenced by the fact that $\widehat{H E D_{k N N}}$ and $\widehat{H E D_{N N}}$ perform significantly worse than $\widehat{H E D} D_{N+}$, if a sufficient number of training instances is provided. This is the case, since $\widehat{H E D} D_{N+}$ can use the provided training data to improve the feature extraction, while $\widehat{H E D_{k N N}}$ and $\widehat{H E D} D_{N N}$ have to rely on the feature extractor, which was pre-trained using the HED. For this reason, $\widehat{H E D} D_{N}+$ performs better than a CNN trained using standard supervised learning if only few labeled samples are provided and similarly well when sufficient training data is available. 
Table II

RECOGNITION ACCURACY IN \% FOR THE EVALUATED RECOGNIZERS ON THE KMNIST DATA SET [6] FOR DIFFERENT TRAINING SET SIZES.

\begin{tabular}{rcccc}
\hline & $\widehat{H E D} D_{k N N}$ & $\widehat{H E D} D_{N N}$ & $\widehat{H E D} D_{N N+}$ & $S$ \\
\hline 10 & $30.90 \pm 2.29$ & $\mathbf{3 1 . 5 1} \pm \mathbf{2 . 0 7}$ & $28.13 \pm 3.03$ & $20.72 \pm 3.16$ \\
30 & $\mathbf{4 4 . 3 5} \pm \mathbf{3 . 7 2}$ & $44.25 \pm 4.74$ & $44.30 \pm 3.96$ & $41.02 \pm 5.27$ \\
50 & $51.47 \pm 2.51$ & $49.01 \pm 2.24$ & $\mathbf{5 2 . 6 4} \pm \mathbf{3 . 7 8}$ & $50.34 \pm 3.16$ \\
70 & $54.54 \pm 3.69$ & $51.17 \pm 3.07$ & $\mathbf{5 6 . 6 8} \pm \mathbf{2 . 8 5}$ & $54.79 \pm 2.69$ \\
100 & $57.57 \pm 3.27$ & $53.98 \pm 1.96$ & $\mathbf{6 2 . 7 9} \pm \mathbf{2 . 9 8}$ & $60.63 \pm 2.42$ \\
250 & $65.23 \pm 2.41$ & $60.08 \pm 1.42$ & $72.29 \pm 2.08$ & $\mathbf{7 2 . 8 4} \pm \mathbf{2 . 6 9}$ \\
500 & $69.42 \pm 1.99$ & $62.25 \pm 0.94$ & $79.43 \pm 1.23$ & $\mathbf{8 0 . 3 1} \pm \mathbf{1 . 3 3}$ \\
1000 & $74.26 \pm 1.49$ & $63.34 \pm 1.13$ & $\mathbf{8 5 . 1 2} \pm \mathbf{0 . 6 6}$ & $84.85 \pm 1.77$ \\
\hline
\end{tabular}

\section{CONCLUSions}

In this paper, we have proposed to use character graphs as privileged information to learn character recognition when only a limited number of labeled data is available for training. We have shown that our approach outperforms a baseline $\mathrm{CNN}$ in cases when only 100 labeled images or less are available for training and equally well if more labeled images are available. We have argued that this loss of advantage in recognition performance, given more training data, is due to the large performance gap between graph matching based approaches to recognition and CNN based approaches, which appears as soon as at least three labeled images per character class are provided. Furthermore, we have shown that our proposed approach performs statistically significantly better than a graph based recognition approach, even if only one labeled sample per class is available. Therefore, it is reasonable to assume that our proposed approach may also perform better than graph matching in other application cases, such as word spotting, in which learning based approaches are currently outperformed by graph matching if only few labeled images are available. Since providing a sufficient amount of labeled data for word spotting tends to be more difficult than for character recognition, the additional processing effort for the proposed pre-training should be more justifiable in this case. Thus, we are planning to investigate the applicability of the proposed approach to word spotting in the future.

\section{ACKNOWLEDGMENT}

This work is part of the research project "Scalable resource-efficient systems for big data analytics" funded by the Knowledge Foundation (grant: 20140032) in Sweden.

\section{REFERENCES}

[1] V. Vapnik, Estimation of dependences based on empirical data. Springer Science \& Business Media, 2006.

[2] V. Vapnik and A. Vashist, "A new learning paradigm: Learning using privileged information," Neural networks, vol. 22, no. 5-6, pp. 544-557, 2009.
[3] D. Doermann, "The indexing and retrieval of document images: A survey," Computer Vision Image Understanding, vol. 70, no. 3, pp. 287-298, 1998.

[4] M. Stauffer, A. Fischer, and K. Riesen, "Keyword spotting in historical handwritten documents based on graph matching," Pattern Recognition, vol. 81, pp. 240-253, 2018.

[5] L. Bottou et al., "Comparison of classifier methods: a case study in handwritten digit recognition," in Proc. 12th IAPR Int. Conf. Pattern Recogn. (ICPR), vol. 2, 1994, pp. 77-82.

[6] T. Clanuwat, M. Bober-Irizar, A. Kitamoto, A. Lamb, K. Yamamoto, and D. Ha, "Deep learning for classical Japanese literature," arXiv preprint arXiv:1812.01718, 2018.

[7] V. Vapnik and R. Izmailov, "Learning using privileged information: similarity control and knowledge transfer." J. Machine Learning Res., vol. 16, no. 2023-2049, p. 2, 2015.

[8] D. Conte, P. Foggia, C. Sansone, and M. Vento, "Thirty years of graph matching in pattern recognition," Int. J. Pattern Recogn. Artificial Intell., vol. 18, no. 03, pp. 265-298, 2004.

[9] P. Foggia, G. Percannella, and M. Vento, "Graph matching and learning in pattern recognition in the last 10 years," Int. J. Pattern Recognition Artificial Intell., vol. 28, no. 01, 2014.

[10] P. Maergner, N. Howe, K. Riesen, R. Ingold, and A. Fischer, "Offline signature verification via structural methods: Graph edit distance and inkball models," in 16th Int. Conf. Frontiers Handwriting Recognition, 2018, pp. 163-168.

[11] A. Fischer, K. Riesen, and H. Bunke, "Improved quadratic time approximation of graph edit distance by combining Hausdorff matching and greedy assignment," Pattern Recognition Letters, vol. 87, pp. 55-62, 2017.

[12] P. Baldi and Y. Chauvin, "Neural networks for fingerprint recognition," Neural Computation, vol. 5, no. 3, pp. 402-418, 1993.

[13] J. Bromley, I. Guyon, Y. LeCun, E. Säckinger, and R. Shah, "Signature verification using a "siamese" time delay neural network," in Advances Neural Inform. Process. Systems, 1994, pp. 737-744.

[14] D. Lopez-Paz, L. Bottou, B. Schölkopf, and V. Vapnik, "Unifying distillation and privileged information," arXiv preprint arXiv:1511.03643, 2015.

[15] Y. Wang, Q. Yao, J. Kwok, and L. M. Ni, "Generalizing from a few examples: A survey on few-shot learning," arXiv preprint arXiv:1904.05046, 2019.

[16] M. Stauffer, A. Fischer, and K. Riesen, "A novel graph database for handwritten word images," in Joint IAPR Int. Workshops Statistical Techn. Pattern Recognition (SPR) Structural and Syntactic Pattern Recognition (SSPR), 2016, pp. 553-563.

[17] K. He, X. Zhang, S. Ren, and J. Sun, "Deep residual learning for image recognition," in Proc. IEEE Conf. Computer Vision Pattern Recognition (CVPR), 2016, pp. 770-778. 